\title{
IMAGES ET TÉLÉVISION : DES SOURCES POUR L'HISTOIRE DU TEMPS PRÉSENT
}

\author{
Marie-Françoise Lévy ${ }^{1}$
}

Les images que la télévision propose, produit et diffuse depuis son lent décollage au lendemain de la Libération ont-elles un lien avec l'histoire ? Et qu'entend-on par histoire ? L'histoire est, ici, comprise comme la marche du temps et le cours des événements dans lesquels s'enracine l'existence des hommes, et l'histoire est également entendue comme discipline scientifique : à ce titre, elle renvoie aux opérations par lesquelles l'histoire se fait, se pense et s'écrit. Poser ces simples repères permet d'éclairer comment la télévision intervient comme acteur de l'histoire à partir des sources propres léguées à l'historien qui cherche à comprendre une période, un temps circonstancié, et à leur donner sens. Cette articulation entre télévision et histoire soulève un ensemble de questions. Elles ont jalonné la rencontre chaotique et sinueuse entre l'histoire -qu'il s'agisse du cours des événements ou de la discipline- et la télévision -comprise comme objet d'études et comme trace du passé.

\footnotetext{
1 IHTP-CNRS.
} 


\section{La levée des obstacles}

Si l'histoire du temps présent ne se fait pas à partir de l'étude d'un matériau unique mais s'élabore dans la confrontation de documents disponibles et différenciés dans leur provenance, cette histoire -en elle-même tension entre un passé proche et un futur inachevé- se fait aussi avec la télévision, ses images et ses usages. Cette approche n'est plus tout à fait neuve : elle s'est construite sur quinze ans, entre 1977 et 1992.

Ce cheminement ne fut pas linéaire. Il aura fallu se déprendre de peurs ou d'inquiétudes, d'une méfiance ou de doutes, d'un rejet et de jugements : autant d'éléments constitutifs de résistances tout d'abord opaques puis explicites enracinant le discrédit de la télévision et des images de télévision avant de parvenir à l'expression d'un questionnement d'ordre scientifique sur les usages de ces sources. Cette reconnaissance, et en quelque sorte la légitimité d'une introduction ou d'une convocation de la télévision et de ses images dans des domaines de recherche est l'aboutissement d'un processus où coexistèrent dans le même temps l'édification de l'histoire du temps présent -ses assises pratiques et théoriques- et l'irruption de nouvelles sources propres à ce passé récent. Or si le cinéma fait l'objet de travaux pionniers -ceux de Marc Ferro et de Pierre Sorlin- publiés en $1977^{1}$ au moment où, précisément, l'histoire du temps présent, controversée, s'expérimente, la télévision quant à elle se situe au seuil de son entrée dans le champ de l'histoire. C'est en effet, en 1977, que Jean-Noël Jeanneney ouvre et dirige avec Monique Sauvage un séminaire sur l'histoire de la télévision à l'Institut d'études politiques de Paris ${ }^{2} .1977$ marque donc une date charnière à partir de laquelle se fondent et l'histoire du temps présent et l'histoire de la télévision dans ses défrichages successifs. Ainsi s'ouvre une période de quinze ans au cours de laquelle se réalisent juxtaposition, ajustements et rencontre entre histoire et télévision.

1 M. Ferro, Cinéma et histoire, Paris, Denoël-Gonthier, 1977 ; P. SOrLiN, Sociologie du cinéma. Ouverture pour l'histoire de demain, Paris, Aubier, 1977.

2 J.-N. JEAnNeney, "Audiovisuel : le devoir de s'en mêler", in J.-P. RrouX et J.-Fr. SIRINELli (dir.), Pour une histoire culturelle, Paris, Éd. du Seuil, 1997, pp. 147163. 
Des obstacles d'ordre moral et culturel ont tout d'abord fait barrage. Diabolique est l'image, mais aussi méprisable est la télévision, cette instance infra-culturelle, cette industrie de programmes et de divertissement qui ne saurait prétendre à la production de connaissances et qui diffuse inlassablement, et sans cohérence apparente, un ensemble confus et composite d'images qui se ressembleraient. Pire encore serait le pouvoir d'attraction, d'envoûtement, de séduction de l'image : celle qui, bien sûr, joue des émotions de celui qui regarde. Et que dire de ces images évanouies chaque soir, de leur caractère éphémère : de leur inexistence. La dimension insaisissable, incernable, des images de télévision a pesé lourdement dans cette histoire du discrédit. Autrement dit ce qu'avaient vu et entendu les Français ne s'incrivait pas dans la durée. Au non sérieux de l'entreprise se mêlent alors, puis se précisent des arguments relevant de l'histoire comme discipline.

La construction négative, oblique, de la représentation des images et de la télévision fut certes un frein majeur à la mise en œuvre d'un travail permettant de passer de la perception d'un flux à la notion de documents produits dans un cadre, un temps, un contexte précis et réalisés par des acteurs identifiables. La levée des obstacles est un mouvement. Y perce la question -légitime- de l'accessibilité aux sources. Ainsi peut-on observer, à partir de ces remarques, que la notion de source et le statut d'une source se construisent selon des critères spécifiques. Trois d'entre eux seront ici retenus :

- la trace matérielle ;

- la constitution de fonds patrimoniaux ;

- l'accessibiité aux documents pour consultation et vérification (mais aussi pour qu'ils puissent être revisités par tout un chacun, historien ou non).

Ces trois conditions ont fait l'objet d'avancées considérables résultant d'un travail des historiens, du législateur et de l'institution (l'Institut National de l'Audiovisuel) dépositaire des œuvres et documents de la radio et de la télévision. La loi sur le dépôt légal du 20 juin 1992 marque, en ce domaine, un tournant fondateur ${ }^{1}$. Cette loi inscrit la reconnaissance du document audiovisuel comme bien

1 M.-F. LÉvy et G. LOCHARD (dir.), "Les sources audiovisuelles du temps présent", MScope, $\mathrm{n}^{\circ} 7$, mai 1994 ; Fr. DENEL, G. PIÉJuT et J.-M. RoDES (dir.), "Le dépôt légal de la radio et de la télévision", Dossiers de l'Audiovisuel, ${ }^{\circ}$ 54, mars-avril 1994. 
patrimonial et comme source de connaissance, elle rend la consultation possible et définit les conditions d'accès aux documents. Ainsi, quinze années furent nécessaires pour que la télévision passe d'un objet insolite à un objet d'investigation et de recherche. Les travaux réalisés au cours de cette période ont très largement contribué au rapprochement entre histoire et télévision. Et si une journée d'études -en hommage à François Bédarida- et une table ronde organisée par le Centre d'histoire de l'Europe du vingtième siècle ${ }^{1}$ - ponctuent en 1992 un temps de bilans et perspectives pour l'histoire du temps présent, la loi sur le dépôt légal votée la même année désigne les chemins de traverse par lesquels la télévision et ses images se sont introduites dans le champ du présent historique lui-même en apprentissage et en définition.

\section{La critique des sources}

L'accès aux documents confère notamment à ces derniers leur statut de source. S'ouvre alors une nouvelle phase où figurent la connaissance de l'histoire de la conservation des fonds, celle de leur organisation comme celle des pratiques documentaires. Elle constituent -en elles mêmes- un ensemble d'informations nécessaires. Comprendre comment les documents sont arrivés jusqu'à nous, connaître la provenance des documents, les diverses voies par lesquelles ils ont été versés, collectés, retrouvés sont autant de renseignements sur le document -sa nature et son statut. Ils en éclairent l'intérêt, la portée et le sens.

Connaître les modes de conservation comme les pratiques d'inventaire et d'indexation permet de distinguer ce que les Français ont vu de l'état de la conservation des documents : d'établir la distinction entre télévision diffusée et télévision conservée. Cette opération donne également des indications sur l'environnement dans lequel s'effectue la fabrication, la réalisation et la diffusion des documents. Ces remarques sur la lecture documentaire des sources tendent à évoquer la mise en perspective d'images s'inscrivant dans une chaîne d'informations contribuant à authentifier et à identifier ces nouveaux

1 Ces deux journées ont donné lieu à des publications : Écrire l'histoire du temps présent. En hommage à François Bédarida, Paris, CNRS Éditions, 1993 ; A. Chauveau et Ph. TÉTART (dir.), Questions à l'histoire des temps présents, Bruxelles, Éd. Complexe, 1992. 
matériaux et supports : à en construire la critique. Les liens entre histoire et télévision se tissent, en effet, par la connaissance et la compréhension des pratiques d'archivage et de conservation des fonds comme par celles des modes et des temps de production et de réalisation des documents de télévision. L'identification des documents passe par cet exercice et les aléas de l'enquête -les absences, les questions en suspens, les ombres, les inconnues : autant d'indices qui tiennent en alerte. Une alerte exacerbée dès lors que l'on prend l'actualité -faits et événements- pour sujets. Ces logiques fluctuantes de fabrication d'images agencées et organisées dans des contextes variables et diversifiés de production constituent donc des outils précieux de connaissance. Ces opérations propres à l'histoire comme discipline sont des palliers successifs qui introduisent la télévision et ses images dans l'histoire -celle du temps présent. La télévision comme instance politique et culturelle et comme vecteur d'informations et de loisirs des Français est fille de son temps. Dans cette perspective, d'autres questions surgissent.

\section{La télévision dans l'histoire}

L'image ment, trompe, déforme, trahit. Elle ne dit pas le vrai. Cette affirmation a priori a contribué au discrédit des sources produites par la télévision. Mais elle engage une réflexion qui consiste à savoir en quoi et pourquoi ces traces sont convoquées. Pour établir les faits ? pour connaitre les formes de transmission des faits et des événements ? pour comprendre ce qu'elles montrent, quand et à qui ? L'image, certes, permet de voir. Cette qualité propre lui confèrerait-telle pour autant un pouvoir ou une vertu exceptionnelle garantissant l'accès à la vérité ? Ces sources valent par ce qu'elles montrent et disent. Les silences, les travestissements, les écarts sont aussi des indices de production non du réel ou du vrai mais d'une réalité : cellelà même qui est donnée à voir : à savoir la transposition et la perception visuelle -volontaire ou non- d'un événément qu'elles ne sauraient, par ailleurs, embrasser dans la totalité. C'est à ce titre que ces images -ces documents- produits par une instance en un temps donné s'inscrivent dans l'histoire : comme acteur de l'histoire. Ainsi peut-on comprendre comment la télévision et ses images prennent sens dès lors qu'elles produisent dans le temps de l'événement -où dans une période délimitée- leurs propres récits et positionnements 
qui sont autant d'actes résultant des événements sur lesquels elles agissent. La télévision et ses images recouvrent des formes diversifiées de participation à l'histoire : auxilliaire politique, instance de régulations, force de mobilisation et de rassemblement, fonction mémorielle, guides de savoir vivre... Autant d'interventions plurielles leur confèrant un rôle d'acteur qui participe de la construction de l'événement et du temps historique dans lesquels elles sont prises.

Les images sont récits, transpositions, figures, interprétations. Ce qui ne saurait pour autant les disqualifier. Les représentations constituent aussi des formes dynamiques de l'histoire et des forces actives d'intervention dans l'histoire. La télévision et ses images, comme traces du passé, et parmi d'autres, concourrent ainsi à l'élargissement de la connaissance du temps présent. 